\title{
Indigo in the nanochannels of zeolite L: Towards a new type of colorant
}

Pascal Woodtli, Sandro Giger, Patrick Müller, Lucie Sägesser, Nicola Zucchetto, Michael J. Reber, Achim Ecker*, Dominik Brühwiler*

* Corresponding Author. E-mail: dominik.bruehwiler@zhaw.ch (DB), achim.ecker@zhaw.ch (AE).

Institute of Chemistry and Biotechnology

Zürich University of Applied Sciences (ZHAW)

CH-8820 Wädenswil

Switzerland

Dyes and Pigments 149 (2018) $456-461$ 


\section{Abstract}

A host-guest based colorant was synthesized by intercalating indigo molecules into the nanochannels of zeolite L (ZL). Reductive washing thereby ensured the efficient and selective removal of nonintercalated indigo molecules. The UV-vis diffuse reflectance spectrum of the product after intercalation and reductive washing (designated as indigo-ZL) was found to resemble the solution spectrum of indigo, leading to the conclusion that the formation of indigo aggregates is prevented due to the steric constraints imposed by the microporous structure of ZL. The application of indigo-ZL on cotton was tested by roll coating. The light absorption properties of the resulting textile prints showed no significant alteration when compared to the pure indigo-ZL powder. The UV-vis diffuse reflectance spectra of mixtures consisting of indigo-ZL and a further ZL-based colorant were successfully predicted by the weighted addition of the respective primary spectra.

Keywords: Indigo; Zeolite; Nanochannel; Host-Guest; Intercalation; Reductive Washing 


\section{Introduction}

Indigo is considered as one of the historically and industrially most relevant colorants [1]. Apart from its widespread use as a blue dyestuff for textiles, indigo is known as the chromophore of the preColumbian pigment Maya Blue [2]. This remarkably stable blue pigment [3] is prepared by incorporating indigo into the pores of the inorganic clay palygorskite. Recent studies have attributed the peculiar hue of Maya Blue pigments to the presence of the oxidized form of indigo (dehydroindigo) [4-6].

The general concept of increasing the stability of an organic molecule by incorporating it into a robust inorganic porous host has been applied to a variety of host-guest combinations. In order to obtain a host-guest material in the strict sense of the word, it is necessary to ensure that the guest species are exclusively present in the host and not on its external surface. Materials fulfilling this requirement are expected to feature well-defined properties, as the guest species are only interacting with the host material, particularly in cases of strong spatial confinement, where even guest-guest interactions can be excluded [7]. Removal of guest species from the external surface of the host - a step that is absent in the synthesis of Maya Blue - is therefore essential regarding the properties of a host-guest material.

In terms of providing host-guest materials with well-defined properties, the use of zeolites as hosts has led to promising results [7]. Zeolites are crystalline aluminosilicates and are based on a network of corner-sharing $\left[\mathrm{SiO}_{4}\right]$ and $\left[\mathrm{AlO}_{4}\right]$ tetrahedra. Following this classical definition, the general chemical formula of a zeolite is represented by $\mathrm{M}_{x / n}\left[\left(\mathrm{AlO}_{2}\right)_{x}\left(\mathrm{SiO}_{2}\right)_{y}\right] \cdot w \mathrm{H}_{2} \mathrm{O}$, where $\mathrm{M}$ is a charge-compensating cation of valence $n$. According to Löwenstein's rule [8], an aluminosilicate cannot contain more $\left[\mathrm{AlO}_{4}\right]$ than $\left[\mathrm{SiO}_{4}\right]$ tetrahedra. A large variety of zeolite frameworks exist, ranging from non-intersecting onedimensional channels to three-dimensionally interconnected cavities [9]. The pore diameter of 
zeolites is in the micropore range (below $2 \mathrm{~nm}$ ). Most zeolites are hydrophilic, except for those with a low content of aluminum. In the context of colorants and fluorescent materials, zeolite L (ZL) has proven to be an excellent host material for organic chromophores and fluorophores [7]. The composition of a unit cell of $\mathrm{ZL}$ is given by $\mathrm{M}_{9 / n}\left[\left(\mathrm{AlO}_{2}\right)_{9}\left(\mathrm{SiO}_{2}\right)_{27}\right] \cdot w \mathrm{H}_{2} \mathrm{O}$. In most cases, $\mathrm{M}$ is a monovalent cation (typically $\mathrm{Na}^{+}$or $\mathrm{K}^{+}$). The amount of $\mathrm{H}_{2} \mathrm{O}$ in a fully hydrated $\mathrm{ZL}$ corresponds to $w=$ 21 [10]. The framework of ZL consists of a hexagonal arrangement of one-dimensional channels with a free diameter of $0.71 \mathrm{~nm}$ at the narrowest and $1.26 \mathrm{~nm}$ at the widest section (Figure 1 ). The centerto-center distance between adjacent channels is $1.84 \mathrm{~nm}$. Crystals of ZL often feature a hexagonal prismatic or approximate cylindrical shape with the channel entrances located on the opposing base surfaces. The size of the crystals can be tuned within a range of $30 \mathrm{~nm}$ to several $\mu \mathrm{m}$ [11]. ZL crystals can furthermore be synthesized with various aspect ratios, including disc, barrel, and rod type morphologies $[11,12]$. For the work reported herein, we have used commercially available ZL crystals with an average diameter of $0.4 \mu \mathrm{m}$ (Figure 1). One such ZL crystal contains approximately 43,000 nanochannels.

Hydroxyl groups on the external surface of the ZL crystals allow for modification by means of functional trialkoxysilanes. A hydrophobic external crystal surface can, for example, be established by reaction with methoxy(dimethyl)octylsilane [13]. External surface functionalization can be employed to optimize the dispersibility in a given medium. This implies that the interaction of a ZL-based hostguest material with its surrounding medium can be tuned independently from the guest species. 


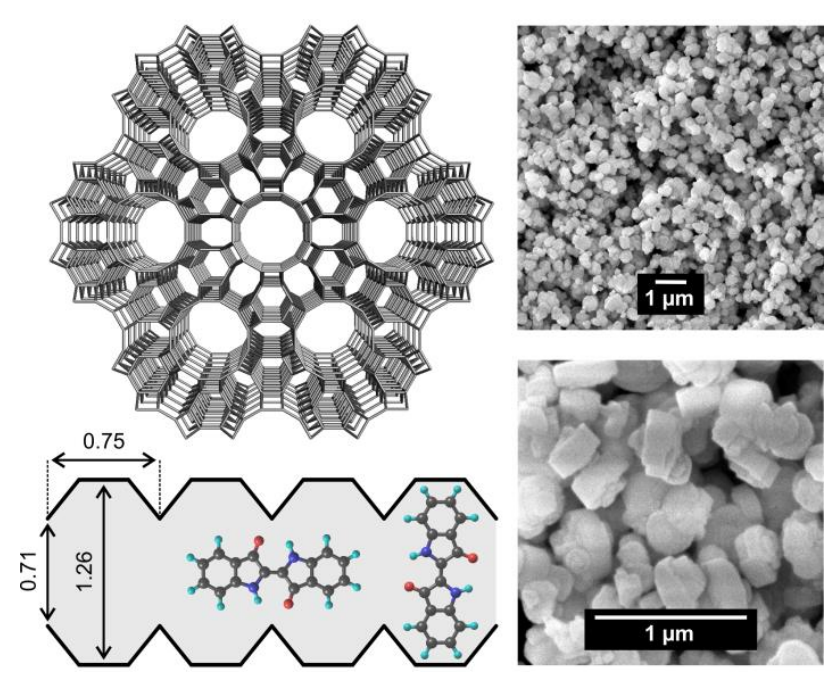

Figure 1. Top left: Framework of ZL viewed along the $c$-axis (channel axis). Bottom left: Illustration of the main channel dimensions (in units of $\mathrm{nm}$ ), compared to the dimensions of the indigo molecule. Right: SEM images of ZL crystals used in this work.

Cationic molecules can be introduced into the channels of ZL from an aqueous solution by means of ion exchange [14]. Loading with neutral molecules is typically conducted by first impregnating the ZL crystals so that the molecule to be introduced into the channels is distributed over the external ZL crystal surface. After drying, the impregnated ZL crystals are heated in a vacuum or under nitrogen to allow for the molecules to diffuse into the channels. Molecules remaining on the external crystal surface are removed by washing with a suitable solvent [15]. Previous work on ZL-based host-guest composites has mainly relied on soluble dyes as guest species. Removing molecules left on the external ZL surface has thus been generally unproblematic, particularly if the ZL channels are sealed before washing to prevent leaching of the intercalated dye molecules $[16,17]$. The intercalation of pigments into ZL is more challenging. Pigments are, by definition, insoluble in common solvents. Alternative ways of removing molecules from the external ZL surface must therefore be developed. 
The host-guest combination of ZL and indigo allows for a new concept of preparing inorganic-organic host-guest materials with well-defined properties. Intercalation and reductive washing leads to an insoluble colorant that - despite of its particulate nature - features the well-defined UV-vis absorption of dissolved indigo molecules.

\section{Materials and Methods}

\subsection{Definition of loading levels}

The target loading level $p_{\mathrm{n}}$ of a guest molecule in $\mathrm{ZL}$ is defined as the average number of guest molecules per unit cell of ZL. To calculate the required amount of indigo for a certain value of $p_{\mathrm{n}}$, a molar mass of a ZL unit cell of $M_{\mathrm{ZL}}=2883 \mathrm{~g} \mathrm{~mol}^{-1}$ was used, corresponding to ZL in its fully hydrated and potassium exchanged state [10]. The actual loading level $p_{\mathrm{n}}{ }^{*}$ is obtained by subtracting the indigo molecules removed upon reductive washing and thus yields the fraction of occupied unit cells according to eq (1).

$p_{\mathrm{n}}^{*}=\frac{\text { number of occupied unit cells }}{\text { total number of unit cells }}$

The mass fraction of indigo $\left(w_{\mathrm{i}}\right)$ in an indigo-ZL sample can then be calculated according to eq $(2)\left(M_{\mathrm{i}}\right.$ $\left.=262.3 \mathrm{~g} \mathrm{~mol}^{-1}\right)$.

$w_{\mathrm{i}}=\frac{p_{\mathrm{n}}^{*} \cdot M_{\mathrm{i}}}{p_{\mathrm{n}}^{*} \cdot M_{\mathrm{i}}+M_{\mathrm{ZL}}}$

A loading of $p_{\mathrm{n}}{ }^{*}=0.5$ therefore corresponds to an indigo content of $4.4 \mathrm{wt} \%$. 


\subsection{Synthesis of indigo-ZL}

ZL (400 g) (HSZ-500, Tosoh Corporation) was dispersed in dry ethyl acetate (1 L). An amount of indigo (Sigma-Aldrich, $95 \%$ ) corresponding to the target loading was added to this suspension and the mixture was sonicated for $20 \mathrm{~min}$. The solvent was subsequently removed by rotary evaporation. The resulting indigo-impregnated ZL sample, designated as indigo@ZL, was heated under reduced pressure (ca. $12 \mathrm{mbar}$ ) to $160{ }^{\circ} \mathrm{C}$. After reaching $160{ }^{\circ} \mathrm{C}$, the vacuum was switched to a nitrogen atmosphere and the temperature was kept for $25 \mathrm{~h}$, leading to the intercalation of the indigo molecules, i.e., to the introduction of the indigo molecules into the $\mathrm{ZL}$ nanochannels. During this process, the powder was stirred.

To remove residual indigo molecules from the external $\mathrm{ZL}$ surface, the indigo-ZL product $(70 \mathrm{~g}$ portions) was dispersed in an aqueous solution (350 mL) of $\mathrm{NaOH}(1 \mathrm{M})$ and $\mathrm{Na}_{2} \mathrm{~S}_{2} \mathrm{O}_{4}(0.05 \mathrm{M})$. After 5 min under quiescent conditions, the suspension was briefly stirred and then centrifuged (10 min, $9000 \mathrm{rpm})$. The yellow supernatant was decanted off and the washing process was repeated until the supernatant was colorless (typically 5 times). The washed blue colored sample, designated as indigoZL, was dispersed in deionized water $(300 \mathrm{~mL})$, centrifuged, and dried under reduced pressure at room temperature. Non-intercalated indigo was recovered by combining the washing solutions containing the yellow leuco-indigo and extracting with cold ethyl acetate, leading to a colorless aqueous phase and a dark blue organic phase. 


\subsection{Synthesis of BY40-ZL}

BY40-ZL can be prepared by a simple ion exchange as previously shown for a variety of cationic dyes [7]. Briefly, BY40 (235 mg) (Basic Yellow 40, CHT Switzerland) was dissolved in water (50 mL) and ZL (10 g) was added. The resulting suspension was stirred at room temperature for $15 \mathrm{~h}$. The product was recovered by centrifugation, washed with four $20 \mathrm{~mL}$ portions of 1-butanol, and dried in a vacuum oven at $80^{\circ} \mathrm{C}$.

\subsection{Channel sealing}

To provide maximum stability against leaching and chemical attack, the channels of ZL can be sealed after intercalation and washing [17]. For this purpose, indigo-ZL (100 g) was dispersed in ethyl acetate (400 mL). While stirring, 3-aminopropyltriethoxysilane (12.5 mL) (APTES, Sigma-Aldrich, $\geq 98 \%$ ) was added. After stirring for $2 \mathrm{~h}$ at room temperature, the suspension was heated to $80^{\circ} \mathrm{C}$ and stirred at this temperature for $16 \mathrm{~h}$. The product was recovered by centrifugation (10 min, $9000 \mathrm{rpm}$ ), washed with ethyl acetate $(7$ times $500 \mathrm{~mL}$ ), and dried under reduced pressure at room temperature.

\subsection{Application by roll coating}

Indigo-ZL and indigo were applied to standard bleached cotton fabric using a commercial printing paste (Printperfekt 226, CHT). In a typical procedure, sealed indigo-ZL (0.5 g) was dispersed in deionized water $(8 \mathrm{~mL})$ for at least $5 \mathrm{~min}$ with an Ultra Turrax mixer (IKA). This dispersion was subsequently mixed with printing paste (50 g, mixing with a turbine agitator for at least $5 \mathrm{~min}$ ). A conventional hand roll coater was used to apply the resulting mixture on cotton. The printed cotton was dried at $80^{\circ} \mathrm{C}$ for $10 \mathrm{~min}$ and cured at $150^{\circ} \mathrm{C}$ for $4 \mathrm{~min}$. When using pure indigo instead of indigo- 
ZL, the amount of colorant in the mixture was adjusted to provide a comparable number of indigo molecules per mass of printing paste. The same procedure was used for applying BY40-ZL (and mixtures of indigo-ZL and BY40-ZL).

\subsection{Physical measurements}

UV-vis diffuse reflectance spectra were obtained with a PerkinElmer Lambda 650 spectrometer equipped with an integrating sphere (150 $\mathrm{mm}$ diameter, with gloss trap). Prior to the measurements, the powder samples were ground in a mortar and pressed to a tablet. Argon sorption isotherms were measured at $87.3 \mathrm{~K}$ with a Quantachrome Autosorb iQ MP equipped with a CryoCooler. Total pore volumes $V_{\text {tot }}$ were derived from the amount of adsorbed argon at a relative pressure of $\mathrm{p} / \mathrm{p}_{0}=0.95$. Samples were dried under vacuum at $120{ }^{\circ} \mathrm{C}$ for at least $3 \mathrm{~h}$ prior to the sorption measurement. Scanning electron microscopy (SEM) images were collected after gold sputtering with a FEI Quanta FEG 250.

\section{Results and Discussion}

\subsection{Reductive washing}

In order to obtain a host-guest material with well-defined and reproducible properties, it is essential to ensure that the amount of guest species adsorbed on the external host surface is minimized. For ZL composites employing soluble dyes as guests, this has been achieved by washing with an appropriate solvent. However, such washing processes pose a challenge due to potential leaching of the intercalated molecules [15]. 
Indigo is insoluble in water, alcohol, and ether, with slight solubility in ethyl acetate, dichloromethane, and DMSO. Removal of externally adsorbed indigo molecules by conventional washing therefore becomes impracticable. The reduction of indigo yields the water soluble leucoindigo (Figure 2). Selective reduction of indigo molecules on the external ZL surface was achieved by using dithionite as a reducing agent. The selectivity of this washing process for the external surface is based on the inability of anions to enter the channels of ZL. The negatively charged ZL framework thus protects the intercalated indigo molecules from attack by the dithionite anion. Figure 2 illustrates the selectivity of the reductive washing by comparing samples before and after intercalation. In the former case, complete reduction of indigo to leuco-indigo occurred, leaving the ZL in its intrinsic colorless form.
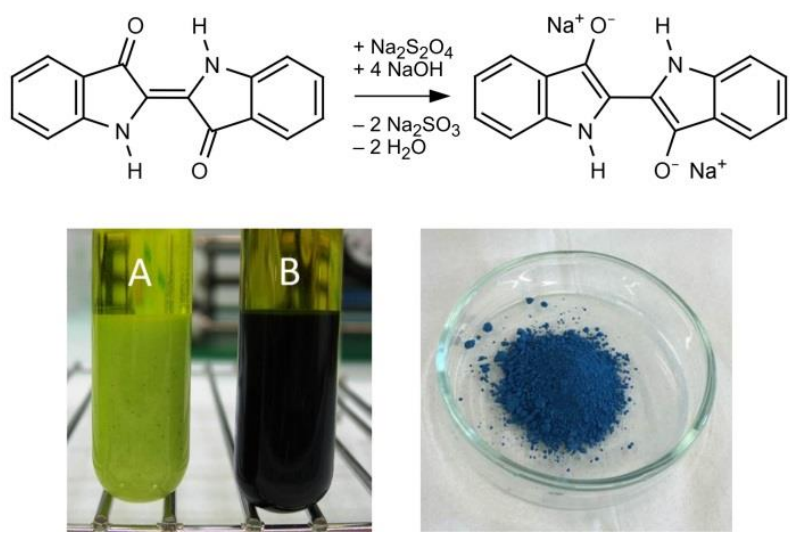

Figure 2. Indigo molecules on the external ZL surface are removed by reductive washing, i.e., by reducing indigo to the water-soluble leuco-indigo (reaction scheme). The photographic image on the left illustrates the outcome of such a reductive washing step when applied to a sample before (test tube A) and after intercalation (test tube B). The image on the right shows a washed and dried indigoZL sample. 


\subsection{Determination of loading levels}

The actual loading level of an indigo-ZL sample can be determined by quantification of the indigo in the washing solutions. After extraction and oxidation (in air), the organic phase was analyzed to determine the concentration of indigo. Figure 3 shows the actual loading level after various intercalation times. Note that at lower temperature, longer intercalation times are required to reach comparable loading levels. After $24 \mathrm{~h}$ at $160{ }^{\circ} \mathrm{C}$, no further significant increase of the loading level could be achieved, indicating that an equilibrium between indigo molecules located on the external ZL surface and indigo molecules located in the ZL nanochannels is reached. In such cases, less than $6 \%$ of the initial amount of indigo was washed off and intercalation was considered to be complete. The actual loading level in this case was $p_{\mathrm{n}}{ }^{*}=0.144$, resulting in an indigo content of $1.3 \mathrm{wt} \%$. For a ZL crystal with a diameter and length of $0.4 \mu \mathrm{m}$, this corresponds to 77 indigo molecules per channel or 3.3 million indigo molecules per ZL crystal.

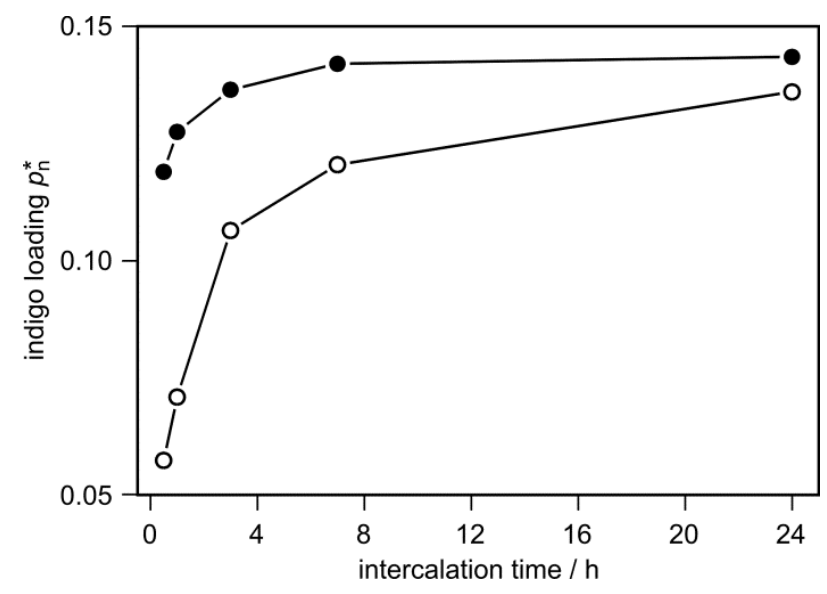

Figure 3. Actual loading levels $p_{\mathrm{n}}{ }^{*}$ as a function of the intercalation time at $120{ }^{\circ} \mathrm{C}$ (open circles) and $160{ }^{\circ} \mathrm{C}$ (solid circles). In both cases the amount of indigo in the starting mixture corresponded to a target loading of $p_{\mathrm{n}}=0.15$. The first data point was taken after $30 \mathrm{~min}$. 


\subsection{Indigo in the ZL nanochannels}

The dimensions of the planar indigo molecule are $0.48 \times 1.2 \mathrm{~nm}^{2}$ [5]. Regarding the orientation of the indigo molecules in the ZL channels, two extreme cases are shown in Figure 1. The diameter of the smallest windows in a ZL channel is $0.71 \mathrm{~nm}$. It is thus reasonable to assume that an indigo molecule roughly aligns its long axis with the ZL $c$-axis (or channel axis) when entering and diffusing through the channels. However, this alignment does not necessarily correspond to the ideal orientation of the molecules in the ZL channels. The long axis of the indigo molecule is slightly shorter than the diameter of the widest channel sections. A situation as shown in Figure 1 with the long axis of indigo perpendicular to the $c$-axis would be possible as well. Due to the very tight fit and the unfavorable host-guest interactions resulting from such an orientation (see below), we consider this particular case unlikely.

The orientation of fluorescent molecules in the channels of ZL has been determined by polarized fluorescence microscopy and was found to strongly depend on the size of the respective molecules [7]. Long-stretched molecules such as perylene diimide (PDI) derivatives typically align with their long axis parallel to the $c$-axis $[17,18]$. The extreme case of perpendicular orientation was observed for $N$ methylacridine, which is $0.15 \mathrm{~nm}$ shorter than indigo $[19,20]$. Due to the very low fluorescence quantum yield of indigo $\left(\Phi_{\mathrm{F}}=0.002\right.$, in solution [21]), it is not possible to reliably determine the orientation of the molecules by means of polarized fluorescence microscopy. Taking into account previous work on differently sized fluorescent dye molecules in ZL [7], it is reasonable to assume that neither of the two cases shown in Figure 1 is an accurate representation of the orientation of indigo molecules in ZL. This becomes evident when considering the possible host-guest interactions. Carbonyl groups are known to favorably interact with the ZL framework $[22,23]$. The indigo molecules will therefore adopt an orientation which maximizes these interactions. This will most likely result in a 
tilt of the molecule's long axis with respect to the $c$-axis. Note that in terms of the interaction between framework and carbonyl groups, the case of perpendicular orientation is unfavorable.

The choice of a host material with a one-dimensional channel system bears several advantages. Compared to a material with a three-dimensional pore system, where every pore entrance provides access to the entire pore volume, materials with one-dimensional channel systems can be sealed more effectively $[16,17,24]$. Not only does this reduce leaching of the guests, but it also limits the diffusion of potentially reactive species into the channels.

The presence of indigo molecules in the ZL channels leads to a significant reduction of the accessible pore volume. Figure 4 shows the argon adsorption isotherms of $\mathrm{ZL}$ and indigo- $\mathrm{ZL}\left(p_{\mathrm{n}}{ }^{*}=0.48\right)$. As expected, ZL displays a type I(a) isotherm, which is generally observed in the case of microporous materials having mainly narrow micropores $(<1 \mathrm{~nm})$ [25]. The argon adsorption capacity is substantially reduced after the intercalation of indigo and reductive washing. A total pore volume of $0.154 \mathrm{~cm}^{3} \mathrm{~g}^{-1}$ was obtained for the pure ZL sample, whereas indigo-ZL $\left(p_{\mathrm{n}}{ }^{*}=0.48\right)$ showed a total pore volume of only $0.037 \mathrm{~cm}^{3} \mathrm{~g}^{-1}$. This is indicative of the strong spatial confinement in indigo-ZL, which - in combination with the one-dimensionality of the ZL channel system - limits diffusion and thus protects the intercalated indigo molecules from chemical attack. Sealing of indigo-ZL with APTES led to a further reduction of the total pore volume $\left(V_{\text {tot }}=0.029 \mathrm{~cm}^{3} \mathrm{~g}^{-1}\right)$. 


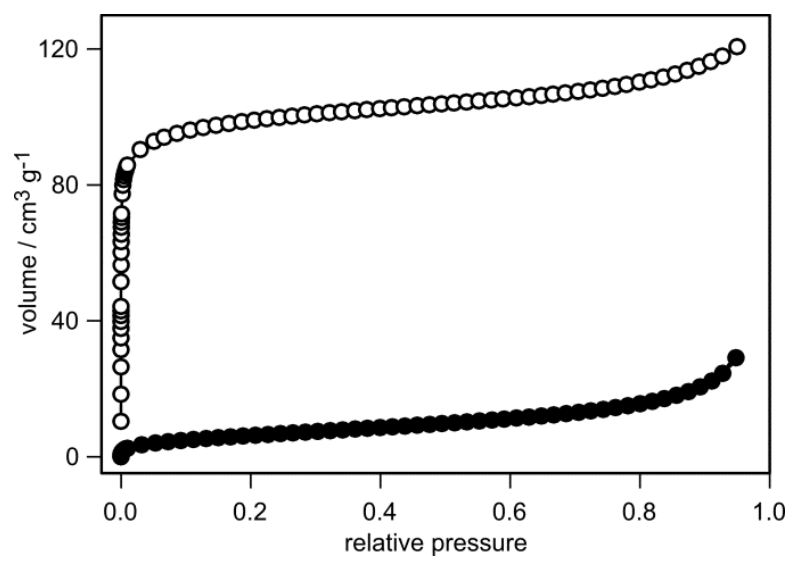

Figure 4. Argon adsorption isotherms of $\mathrm{ZL}$ (open circles) and indigo-ZL $\left(p_{\mathrm{n}}{ }^{*}=0.48\right.$, before sealing with APTES, solid circles).

\subsection{UV-vis spectroscopy of indigo-ZL}

Figure 5 (panel A) compares the UV-vis diffuse reflectance spectra of a mechanical mixture of indigo and ZL with the spectra of indigo@ZL (impregnated) and indigo-ZL (after intercalation and washing). The spectrum of the mechanical mixture features the well-established spectrum of solid indigo, which has a broad maximum around $650 \mathrm{~nm}$ [4]. A new band with a maximum at $620 \mathrm{~nm}$ appears after impregnation, indicating partial intercalation of the indigo molecules. A narrow absorption band with a maximum at $615 \mathrm{~nm}$ is ultimately obtained after intercalation and washing. Although slightly broader, this spectrum is very similar to the solution spectrum of indigo in DMSO (Figure 5, panel B). The bathochromic shift of the absorption maximum of solid indigo with respect to indigo in organic solvents has been attributed to hydrogen bonding between the indigo molecules in the solid state $[26,27]$. We can thus conclude that the indigo molecules in the channels of ZL are predominantly present in the form of monomers. The spectrum of indigo-ZL differs significantly from spectra of 
classical Maya Blue pigments. The latter are generally much broader due to variable contributions from indigo aggregates, indigo monomers, and dehydroindigo [4].

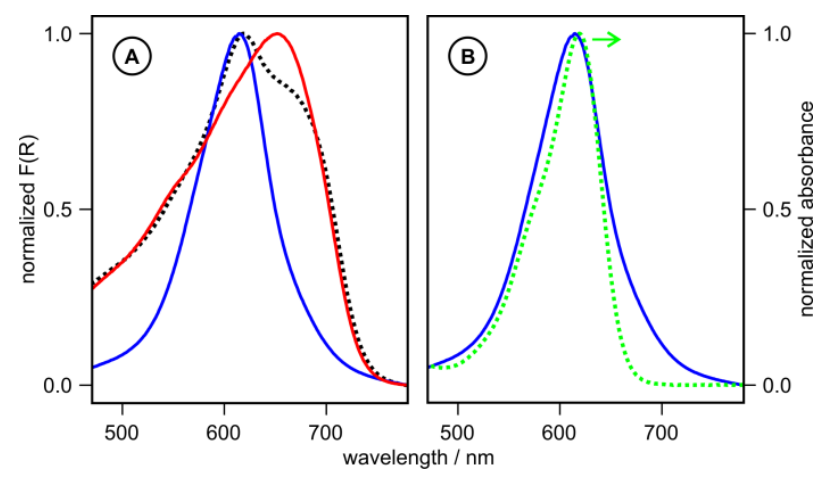

Figure 5. Normalized diffuse reflectance spectra of a mechanical mixture of indigo and ZL (panel A, red), indigo@ZL (panel A, black dots), and washed indigo-ZL (panel A, blue). The diffuse reflectance spectrum of washed indigo-ZL (blue) is compared to the solution spectrum of indigo in DMSO (green dots) in panel $\mathrm{B}$. The actual loading level of the washed indigo-ZL sample is $p_{\mathrm{n}}{ }^{*}=0.48$.

To exemplify that the narrow absorption band of indigo-ZL is independent of the application medium, we have prepared indigo-ZL prints on cotton by means of a commercial printing paste. Figure 6 shows that the diffuse reflectance spectra of the resulting prints remain comparable to the solution spectrum of indigo. Apart from a slight broadening at high loading levels, presumably indicative of minor intermolecular interactions in the ZL channels due to the shorter distances between the indigo molecules, the shape of the indigo-ZL absorption band is largely independent of the loading level (Figure 6, panel A). It should be mentioned at this point that a comparison between the color characteristics of indigo-ZL and Maya Blue is not straightforward. The hue of Maya Blue is affected by the indigo/dehydroindigo ratio, which in turn is determined by the thermal treatment during the 
preparation of the pigment $[2,4]$. In the case of indigo-ZL, intercalation under an inert atmosphere (excluding aerobic oxidation of indigo) and reductive washing ensure a product with a well-defined hue.

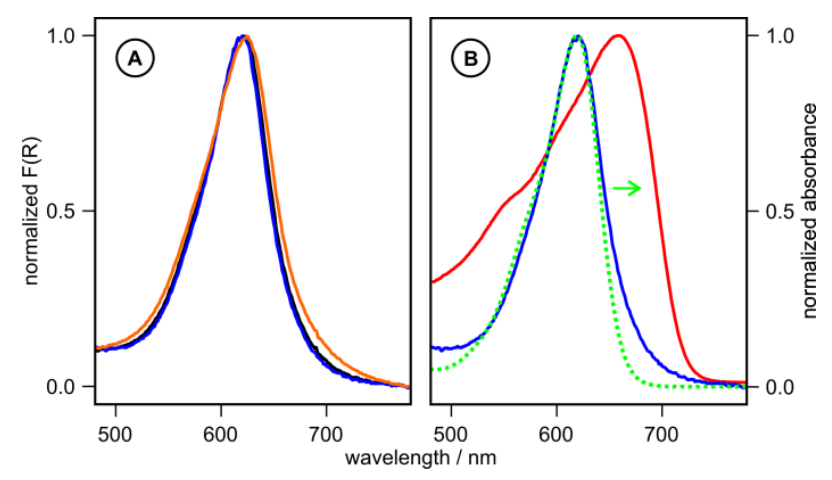

Figure 6. Normalized diffuse reflectance spectra of indigo-ZL textile prints with loading levels of 0.5 (panel A, orange), 0.25 (panel A, black), and 0.15 (panel A, blue). Panel B compares the indigo-ZL textile print (blue, $p_{\mathrm{n}}=0.15$ ) to a textile print prepared with pure indigo (red). The solution spectrum of indigo in DMSO (green dots) is shown for comparison.

Regarding the use of ZL-based host-guest materials as colorants, it should be noted that the refractive index of ZL is 1.49 [28] and thus similar to the refractive index of common organic polymers (or conventional organic pigments). The application of ZL-based colorants in such polymer matrices (e.g. for coatings) typically leads to low opacity [29]. 


\subsection{Mixing behavior}

The chemical and physical properties of a pigment, such as surface functional groups, particle size and shape, as well as crystal modifications must be taken into account when considering potential applications [30]. When using porous materials to host the chromophores, the essential chemical and physical properties of the pigment - apart from light absorption - are determined by the porous material, and are therefore independent of the color. It follows that once a process has been found to apply a certain ZL-based colorant in a specific medium, all other ZL-based colorants can be applied by the same process. Furthermore, we can expect an ideal mixing behavior, implying that the diffuse reflectance spectrum of a mixture of ZL-based colorants should be predictable by the weighted addition of the respective primary spectra. To test this hypothesis we have investigated mixtures of indigo-ZL and BY40-ZL. As expected, dispersion of the bright yellow BY40-ZL in the printing paste was successfully achieved by the procedure used for indigo-ZL. Figure 7 shows the experimental diffuse reflectance spectrum of a textile print obtained with an indigo-ZL/BY40-ZL mixture. The spectrum is in good agreement with the spectrum calculated by the weighted addition of the indigo-ZL and BY40-ZL primary spectra. 

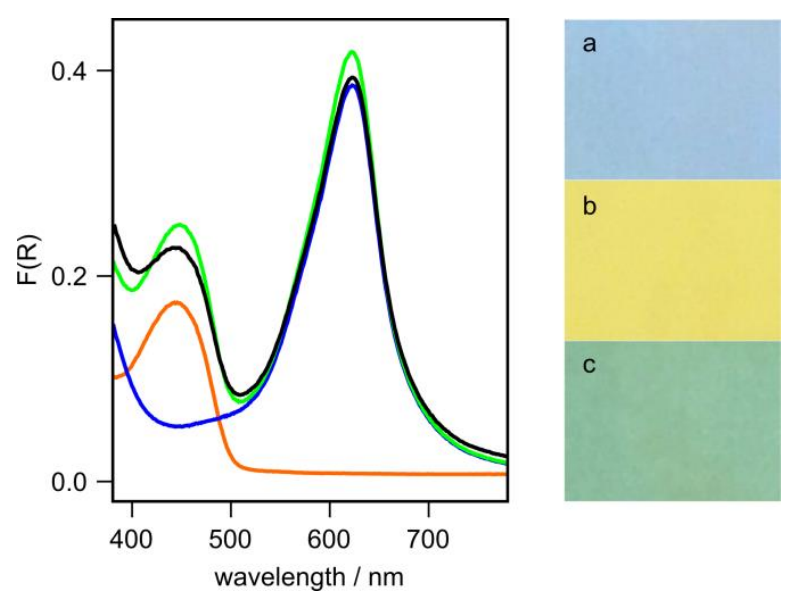

Figure 7. Diffuse reflectance spectra and photographic images of textile prints prepared with indigoZL (blue line, image a), BY40-ZL (orange line, image b), and an indigo-ZL/BY40-ZL mixture (green line, image c). The spectrum obtained by adding the indigo-ZL and BY40-ZL spectra is shown in black.

\section{Conclusions}

Pigments are defined as colorants that are insoluble in the application medium, implying that they retain a particulate (or crystal) structure throughout the coloration process [30]. According to this definition, indigo-ZL can be considered as a pigment, although its light absorption properties are comparable to dissolved indigo monomers. By preventing the formation of indigo aggregates, the absorption spectrum of indigo-ZL in a matrix or on a substrate is highly predictable, i.e., it will in any case resemble the indigo solution spectrum.

The reproducibility of the UV-vis diffuse reflectance spectra in different application media requires the indigo molecules to be shielded. It is therefore essential to remove indigo molecules that are adsorbed on the external ZL surface. The method of reductive washing ensures a high selectivity for indigo molecules on the external ZL surface and enables recycling of the non-intercalated indigo molecules. 
The hue of conventional organic pigments depends not only on the light absorption properties of the chromophores, but is to a large extent determined by the particle size and crystallinity [27]. The hostguest principle applied for the synthesis of indigo-ZL opens possibilities for adjusting the particle size of a pigment without significantly affecting its light absorption properties. Furthermore, by using different chromophores, a range of pigments can be prepared that are identical in terms of particle size, particle morphology, dispersibility, and surface chemistry.

Acknowledgement. Financial support by the Swiss National Science Foundation (project 20PC21_161557) and the Commission for Technology and Innovation (project 18622.1 PFNM-NM) is acknowledged. 


\section{References}

[1] Ferreira ESB, Hulme AN, McNab H, Quye A. The natural constituents of historical textile dyes. Chem Soc Rev 2004;33:329-36.

[2] Doménech A, Doménech-Carbó MT, Sánchez del Rio M, Vázquez de Agredos Pascual ML, Lima E. Maya Blue as a nanostructured polyfunctional hybrid organic-inorganic material: the need to change paradigms. New J Chem 2009;33:2371-79.

[3] Fois E, Gamba A, Tilocca A. On the unusual stability of Maya blue paint: molecular dynamics simulations. Microporous Mesoporous Mater 2003;57:263-72.

[4] Rondão R, Seixas de Melo JS, Bonifácio VDB, Melo MJ. Dehydroindigo, the forgotten indigo and its contribution to the color of maya blue. J Phys Chem A 2010;114:1699-708.

[5] Tilocca A, Fois E. The color and stability of Maya blue: TDDFT calculations. J Phys Chem C 2009;113:8683-87.

[6] Doménech A, Doménech-Carbó MT, Vázquez de Agredos Pascual ML. Dehydroindigo: a new piece into the Maya Blue puzzle from the voltammetry of microparticles approach. J Phys Chem B 2006;110:6027-39.

[7] Brühwiler D, Calzaferri G, Torres T, Ramm JH, Gartmann N, Dieu LQ, Lopez-Duarte I, Martinez-Diaz MV. Nanochannels for supramolecular organization of luminescent guests. J Mater Chem 2009;19:8040-67.

[8] Loewenstein W. The distribution of aluminum in the tetrahedra of silicates and aluminates. Am Mineral 1954;39:92-6.

[9] Baerlocher C, McCusker LB, Olson DH. Atlas of zeolite framework types. 6th ed. Amsterdam: Elsevier; 2007.

[10] Barrer RM, Villiger H. The crystal structure of the synthetic zeolite L. Z Kristallogr 1969;128:352-70.

[11] Zabala Ruiz A, Brühwiler D, Ban T, Calzaferri G. Synthesis of zeolite L: Tuning size and morphology. Monatsh Chem 2005;136:77-89.

[12] Lupulescu Al, Kumar M, Rimer JD. A facile strategy to design zeolite L crystals with tunable morphology and surface architecture. J Am Chem Soc 2013;135:6608-17.

[13] Bossart O, Calzaferri G. Organisation and solubilisation of zeolite L crystals. Chimia 2006;60:179-81.

[14] Gfeller N, Calzaferri G. Thionine in the cage of zeolite L. J Phys Chem 1992;96:3428-35.

[15] Brühwiler D, Gfeller N, Calzaferri G. Resorufin in the channels of zeolite L. J Phys Chem B 1998;102:2923-9.

[16] Ban T, Brühwiler D, Calzaferri G. Selective modification of the channel entrances of zeolite $L$ with triethoxysilylated coumarin. J Phys Chem B 2004;108:16348-52. 
[17] Cao P, Khorev O, Devaux A, Sägesser L, Kunzmann A, Ecker A, Häner R, Brühwiler D, Calzaferri G, Belser P. Supramolecular organization of dye molecules in zeolite L channels: Synthesis, properties, and composite materials. Chem Eur J 2016;22:4046-60.

[18] Tabacchi G, Calzaferri G, Fois E. One-dimensional self-assembly of perylene diimide dyes by unidirectional transit of zeolite channel openings. Chem Comm 2016;52:11195-8.

[19] Huber S, Zabala Ruiz A, Li H, Patrinoiu G, Botta C, Calzaferri G. Optical spectroscopy of inorganic-organic host-guest nanocrystals organized as oriented monolayers. Inorg Chim Acta 2007;360:869-75.

[20] Fois E, Tabacchi G, Devaux A, Belser P, Brühwiler D, Calzaferri G. Host-guest interactions and orientation of dyes in the one-dimensional channels of zeolite L. Langmuir 2013;29:9188-98.

[21] Seixas de Melo J, Moura AP, Melo MJ. Photophysical and spectroscopic studies of indigo derivatives in their keto and leuco forms. J Phys Chem A 2004;108:6975-81.

[22] Calzaferri G, Brühwiler D, Meng T, Dieu L-Q, Malinovskii VL, Häner R. Surprising properties of a furo-furanone. Chem Eur J 2010;16:11289-99.

[23] Devaux A, Minkowski C, Calzaferri G. Electronic and Vibrational Properties of Fluorenone in the Channels of Zeolite L. Chem Eur J 2004;10:2391-408.

[24] Tsotsalas MM, Kopka K, Luppi G, Wagner S, Law MP, Schäfers M, De Cola L. Encapsulating ${ }^{111}$ In in nanocontainers for scintigraphic imaging: synthesis, characterization, and in vivo biodistribution. ACS Nano 2010;4:342-48.

[25] Thommes M, Kaneko K, Neimark AV, Olivier JP, Rodriguez-Reinoso F, Rouquerol J, Sing KSW. Physisorption of gases, with special reference to the evaluation of surface area and pore size distribution (IUPAC Technical Report). Pure Appl Chem 2015;87:1051-69.

[26] Monahan AR, Kuder JE. Spectroscopic differences between crystalline and amorphous phases of indigo. J Org Chem $1972 ; 37: 4182-84$

[27] Thetford D, Cherryman J, Chorlton AP, Docherty R. Theoretical molecular modelling calculations on the solid state structure of some organic pigments. Dyes and Pigments 2004;63:259-76.

[28] Busby M, Blum C, Tibben M, Fibikar S, Calzaferri G, Subramaniam V, De Cola L. Time, space, and spectrally resolved studies on J-aggregate interactions in zeolite L nanochannels. J Am Chem Soc 2008;130:10970-76.

[29] Bauer C, Gartmann N, Dieu L-Q, Zuber N, Dolamic I, Ramm JH, Brühwiler D. Self-organized patterns of microparticles in polymer films. Thin Solid Films 2011;519:3674-78.

[30] Hunger K, Herbst W. Pigments, organic. In: Ullmann's Encyclopedia of Industrial Chemistry, Wiley-VCH; 2000, Vol. 27, p. $379-423$. 